\title{
Computed Tomography of an Anatomical Site of Interest without Contrast
}

National Cancer Institute

\section{Source}

National Cancer Institute. Computed Tomography of an Anatomical Site of Interest

without Contrast. NCI Thesaurus. Code C137901.

Computed tomography of an anatomical site of interest without the use of a contrast agent. 